\title{
Bilateral Ptosis with Hypoglossal Nerve Palsy in Guillain-Barre Syndrome: A Rare Presentation
}

Jayitri Mazumdar*, Tunisha Bhattacharya, Chandan Kumar Sing and Mousumi Nandi

Department of Pediatrics, Calcutta National Medical College and Hospital, Kolkata, India

\begin{abstract}
Cranial nerve involvement in Gullian-barre syndrome (GBS) is common and a well-known fact, however there are few studies only focusing on cranial nerve involvement in GBS. We here describe a 12 yrs old boy presented with flaccid quadruparesis and respiratory distress. There was inability to elevate both the upper eyelids, deviation of tongue to the right and deviation of angle of mouth to the left. The patient was treated with IVIG $(2 \mathrm{mg} / \mathrm{kg})$ and was ventilated in volume control mode for 10 days and then tracheostomy tube was inserted, which was removed after 2 wks. Patient achieved complete recovery of all 4 limbs with no residual neurodeficit. Though cranial nerves are involved frequently in GBS but bilateral $3^{\text {rd }}$ nerve involvement manifesting as bilateral ptosis and $12^{\text {th }}$ nerve involvement are very rare till date and if managed early with IVIG the recovery is remarkable in pediatric age group.
\end{abstract}

Keywords: Guillain-Barré syndrome; Cranial nerve palsy

\section{Introduction}

Gullian-Barré syndrome (GBS) is an acute inflammatory polyradiculoneuropathy, characterized by a rapidly progressive, symmetric, Areflexic flaccid paralysis with albumin-cytological dissociation in CSF [1]. Proximal and distal muscles are involved relatively symmetrically, but asymmetry is found in $9 \%$ of patients. The onset is gradual and progresses over days or weeks; the process plateaus in 1-28 days. Particularly in cases with an abrupt onset, tenderness on palpation and pain in muscles are common in the initial stages. Affected children are irritable. Weakness can progress to inability or refusal to walk and later to flaccid tetraplegia. Maximal severity of weakness is usually reached by 4 week after onset. Bulbar involvement occurs in about half of cases. Miller-Fisher syndrome (MFS) consists of acute external and occasionally internal opthalmoplegia, ataxia, and areflexia. The $6^{\text {th }}$ cranial nerve is most often involved in MFS. Papilledema may precede or follow MFS and suggests a diagnosis of pseudo tumor; optic neuritis may also be noted [2]. Motor nerve conduction velocities are greatly reduced and sensory nerve conduction time is often slow. Electromyography shows evidence of acute denervation of muscle. Serum creatinine kinase levels may be mildly elevated or normal. Antiganglioside antibodies against GQ1b are sometimes elevated in serum particularly in miller-fisher variant. Serologic testing for campylobacter and helicobacter infection helps in establishing the cause if results are positive but does not alter course of treatment [2].

In a study by Bhargava et al cranial nerve involvement was seen in $(62.3 \%)$ patients [1]. Many of the studies done before quote variable involvement of cranial nerves ranging from $50 \%$ to $75 \%$, for example, L $\varphi$ effel, et al [3] have quoted $50 \%$ and Dhadke et al. [4] had $62.5 \%$ involvement. Bulbar palsy was the most common $(49.2 \%)$ in majority of the studies [3]. But bilateral $3^{\text {rd }}$ nerve palsy manifesting as ptosis along with hypoglossal nerve palsy is a rare association in childhood GBS.

Case: 12 years old male, was admitted in this hospital with chief complaints of Inability to move all 4 limbs for last 7 days, Respiratory distress for last 4 days and inability to sustain normal respiration for last $24 \mathrm{hrs}$. The illness started with fever which was acute in onset, low grade, intermittent in nature. Patient was alert and conscious. Fever subsided after 3 days, followed by loss of power in both the lower limbs and deviation of angle of mouth to left with inability to open both eyes. Not associated with any rash, bleeding manifestation, headache, vomiting, convulsion, blurring of vision, the weakness was progressive and ascending in nature. Inability to sustain normal respiration has progressed to severe respiratory distress in a course of 7 days. Associated with urinary retention. No fecal incontinence. Pt complained deviation of angle of mouth to the left with inability to open both eyes. There was no history of dog bite or recent vaccination. On examination there was absence of upward movement of both eyelids (bilateral ptosis). Paraplegia with hypotonia in both lower limbs, absent deep tendon reflexes and planter equivocal, power $4 / 5$ in both upper limbs, $2 / 5$ in both lower limbs. There was associated urinary retention. There was no sensory loss. Deviation of angle of mouth to the left (right sided VII nerve palsy). Surprisingly the tongue was deviated to the right when protruded. (Right sided XII nerve palsy) (Figure 1 and 2). Laboratory investigations showed, $\mathrm{Hb} 11.4 \mathrm{gm} / \mathrm{dl}$, TLC $7500 / \mathrm{cmm}$, Platelet 1,40,000/cmm. Urea: 26 Cr 0.6, Na 142, K 3.5, LFT WNL. CSF study: clear, pressure not raised, cell count nil, protein $160 \mathrm{mg} / \mathrm{dl}$, albumino cytological dissociation, sugar $51 \mathrm{mg} / \mathrm{dl}$, chloride $118 \mathrm{mg} / \mathrm{dl}$. Gram stain, AFB stain negative. MRI brain and spine were within normal limit. NCV of all 4 limbs showed features of AMAN (acute motor axonal type of neuropathy) (Figure 3 and 4). EMG showed denervation pattern (Figure 5).

Patient was treated with IVIG $(2 \mathrm{mg} / \mathrm{kg})$ and was ventilated in volume control mode for 10 days and then after patient's trigger for respiration came he was extubated after keeping for 2 days in weaning mode and then tracheostomy tube was inserted, as the patient could not clear his secretions and the tube was removed after 2 wks.Patient achieved complete recovery of power in all 4 limbs with no residual neurodeficit. As the patient was on ventilator for initial 10 days

*Corresponding author: Jayitri Mazumdar, Department of Pediatrics, Calcutta National Medical College West Bengal University of Health Sciences, Kolkata India, Tel: +91-9230297392; Fax: +91-9222843582; E-mail: jayidoc@gmail.com

Received September 16, 2015; Accepted October 26, 2015; Published October 30, 2015

Citation: Mazumdar J, Bhattacharya T, Sing CK, Nandi M (2015) Bilateral Ptosis with Hypoglossal Nerve Palsy in Guillain-Barre Syndrome: A Rare Presentation. J Neurol Neurophysiol 6: 320. doi:10.4172/2155-9562.1000320

Copyright: (c) 2015 Mazumdar J, et al. This is an open-access article distributed under the terms of the Creative Commons Attribution License, which permits unrestricted use, distribution, and reproduction in any medium, provided the original author and source are credited. 
Citation: Mazumdar J, Bhattacharya T, Sing CK, Nandi M (2015) Bilateral Ptosis with Hypoglossal Nerve Palsy in Guillain-Barre Syndrome: A Rare Presentation. J Neurol Neurophysiol 6: 320. doi:10.4172/2155-9562.1000320

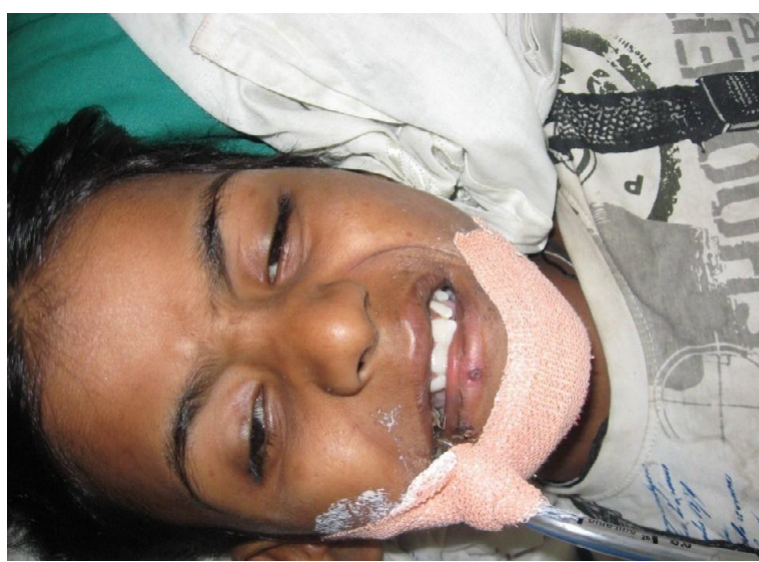

Figure 1: Showing right sided $\mathrm{VI}^{\text {th }}$ nerve palsy.

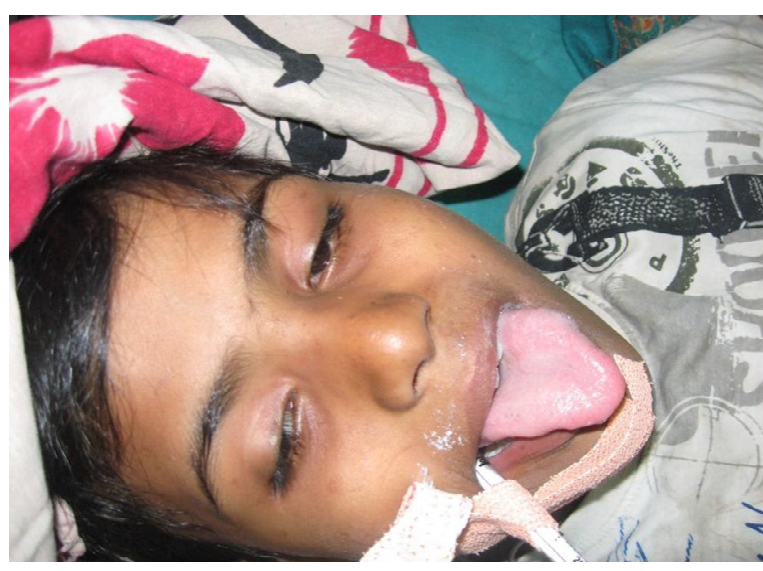

Figure 2: Showing bilateral ptosis and deviation of tongue to the right.

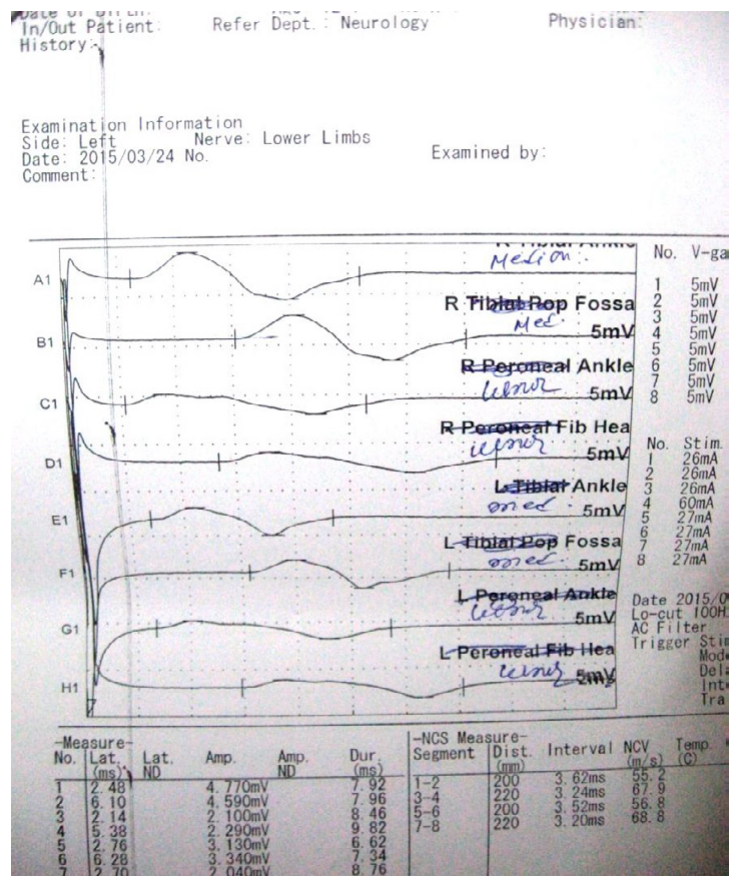

Figure 3: Showing increased distal CMAP (compound motor action potential) latency in upper limb.

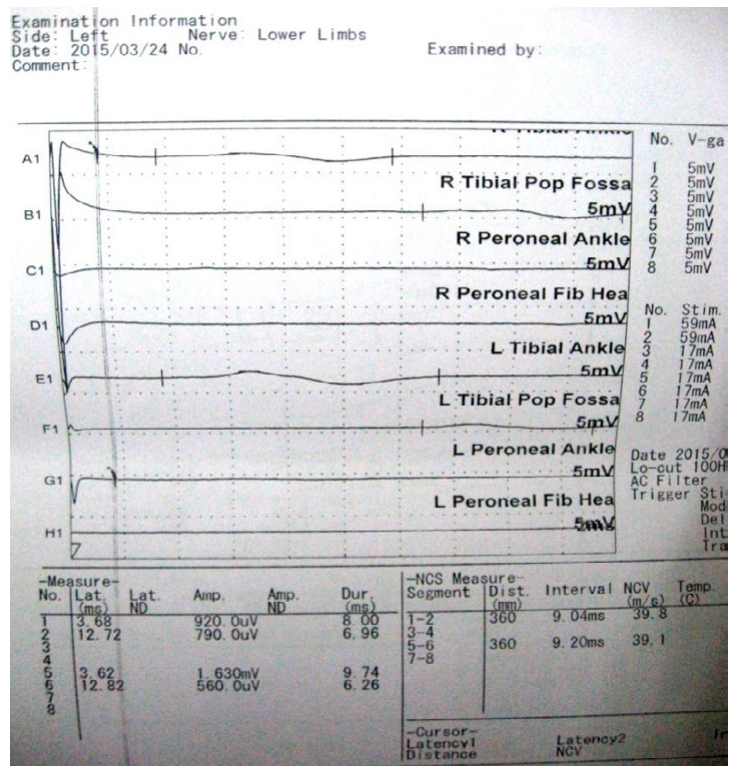

Figure 4: Showing increased distal CMAP (compound motor action potential) latency in lower limb.

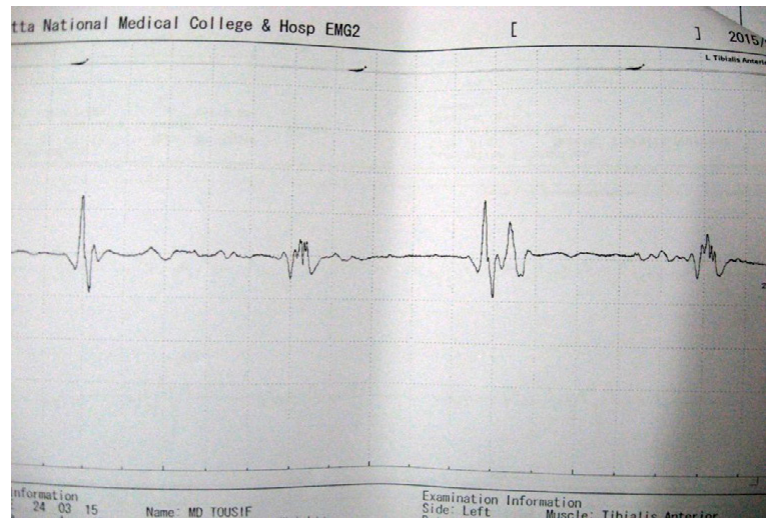

Figure 5: EMG of lower limb showing denervation pattern.

electrophysiological study for blink reflex and facial nerve EMG could not be done. Later, during extubation the ptosis, facial palsy and tongue deviation improved leaving only appreciable weakness in all 4 limbs. So we did EMG and NCV of all 4 limbs which revealed AMAN (acute motor axonal neuropathy). As the patient did not show any feature of Miller-Fisher syndrome (MFS) we did not go for any serological testing for GQ1b antibody specific for MFS.

\section{Discussion}

Gullian-Barrie syndrome can present with cranial nerve involvement and bulbar palsy. Bulbar involvement occurs in about half of cases [1]. Dysphagia and facial weakness are often impending signs of respiratory failure. They interfere with eating and increase the risk of aspiration. The facial nerves may be involved. Some young patients exhibit symptoms of viral meningitis or meningoencephalitis. Extra ocular muscle involvement is rare, but in an uncommon variant, oculomotor and other cranial neuropathies are severe early in the course [2]. $3^{\text {rd }}$ nerve palsy with only manifestation being bilateral ptosis is not reported so far in medical literature. Unilateral XII nerve palsy 
Citation: Mazumdar J, Bhattacharya T, Sing CK, Nandi M (2015) Bilateral Ptosis with Hypoglossal Nerve Palsy in Guillain-Barre Syndrome: A Rare Presentation. J Neurol Neurophysiol 6: 320. doi:10.4172/2155-9562.1000320

is also very rare. It is only reported in some adult patients with GB syndrome.

In the study by Bhargava et al. Hypoglossal nerve involvement was seen in six (10\%) patients and four (6.5\%) patients had opthalmoplegia [1]. Whereas in the study by Dhadke, et al hypoglossal nerve involvement was $0 \%$. Reports are rare in pediatric age group. Facial nerve was involved in 12 [30\%] patients and Glossopharyngeal, vagus nerves were involved in $12[30 \%]$ patients ${ }^{4}$.Madoori et al has reported a case of GBS with $9^{\text {th }}, 10^{\text {th }}, 11^{\text {th }}, 12^{\text {th }}$ cranial nerves in a 6 years old boy [5].

Most children experience full recovery, some may have residual defects. Bad prognosis is associated with maximum disability at presentation, respiratory muscle paralysis needing intubation, multiple cranial nerve involvement ${ }^{1}$. Surprisingly our patient achieved full recovery inspite of having respiratory involvement along with multiple cranial nerve involvement. So the purpose of this case report is not only to cite the uncommon presentation but also to convey the message that full recovery is possible with early and aggressive treatment in GBS with maximum disability at presentation.

\section{References}

1. Bhargava A, Banakar BF, Pujar GS, Khichar S (2014) A study of Guillain-Barré syndrome with reference to cranial neuropathy and its prognostic implication. $J$ Neurosci Rural Pract 5: 43-47.

2. Kliegman, Stanton, St Geme, Schor (2015) Gullain Barre Syndrome. Textbook of Pediatrics; (20thedn), Saunders Elsevier, Philadelphia, London.

3. Löffel NB, Rossi LN, Mumenthaler M, Lütschg J, Ludin HP (1977) The LandryGullian Barré syndrome. Complications, prognosis and natural history in 123 cases. J Neurol Sci 33:71-79.

4. Dhadke SV, Dhadke VN, Bangar SS, Korade MB (2013) Clinical profile of Guillain Barre Syndrome. J Assoc Physicians India 61: 168-172.

5. Madoori S, Sunitha L, Radhika K, Ramya MY (2014) Bulbar Guillain-Barre Syndrome, International Journal of Medical and Applied Sciences 3: 202-204. 